\title{
Therapeutic Potential of Selenium Nanoparticles
}

\author{
Warda Majeed, Maryam Zafar, Attya Bhatti* and Peter John
}

National University of Sciences and Technology (NUST), Sector H-12, Islamabad, Pakistan

\begin{abstract}
Selenium plays essential role in regulating function of many selenoproteins in body. It is an important micronutrient and its supplementation provides tremendous health benefits. It has been found that humans have 25 selenoproteins that play important roles in the body. Therefore, deficiency of selenium can lead to many chronic diseases such as rheumatoid arthritis. Selenium is also important in reducing inflammation, decreasing oxidative stress and in improving bone health. Selenium improves functioning of immune system, maintains oxidative balance in body and decreases bone resorption. Toxicicity issues related to selenium have moved the direction of research towards nano particles (NPs) of selenium as in nano form selenium is less toxic and safer for living systems. In nano form the entire properties of selenium are changed as compared to its bulk form. In this review, we have described the various health-care applications of SeNPs (selenium nanoparticles). It is also discussed that how nanoselenium can act as a promising agent in treating chronic health disorders.
\end{abstract}

Keywords: Nanotechnology; Selenoproteins; Antioxidant activity; Bone health; Toxicity

\section{Introduction}

Selenium is an important trace element, it was previously considered toxic but a decade ago researchers proved its importance to the human health [1]. The function of selenium in body is achieved by its incorporation into selenoproteins as selenocysteine, $21^{\text {th }}$ amino acid at active site of selenoproteins [2]. The UGA codon in mRNA allows the incorporation of selenocysteine into selenopoteins under specific conditions along with other factors. Importance of selenoproteins to human health has been identified by single nucleotide polymorphisms (SNPs) in genes encoding these proteins [3]. In mammals, at least 30 selenoproteins have been identified and it has been found that humans have 25 selenoproteins that play important roles in the body [2]. Therefore, deficiency of Se leads to severe health disorders like Rheumatoid Arthritis (RA). A number of studies have been conducted to determine the status of selenium in patients of rheumatoid arthritis and it was found that concentration of Se (selenium) was low in serum and synovial fluid of RA patients [4,5]. Rheumatoid arthritisis a chronic, progressive, inflammatory autoimmune disease that is described by synovial joint pain, swelling and tenderness which ultimately leads to disability and short life span [6]. RA effects 0.5 to $1 \%$ of the population globally. Genetic and environmental factors both contributes to progression of RA [7]. The well-known genetic risk factor for RA is HLA locus that accounts for 30-50 percent of the overall genetic susceptibility to RA [8]. Some clinical trials showed anti-arthritic potential of selenium while others do not. An open trial with $160 \mathrm{ug}$ of selenite as supplement in ten RA patients had positive effect but a double blind study on active RA patients with 256 ug of yeast selenium produced no anti-inflammatory effects [9]. The common forms of selenium in foods are selenomethionine, selenocysteine, selenoneine (2-selenyl-Na,Na,Na-trimethyl-L-histidine), Se-methylselenocysteine and $\gamma$-glutamyl-Se methylselenocysteine, Sodium selenite and selenite [10]. In USA the dietary selenium intake ranges from from $7 \mu \mathrm{g}$ per day to $4990 \mu \mathrm{g}$ per day, with mean values of $40 \mu \mathrm{g}$ per day in Europe and 93 $\mu \mathrm{g}$ per day (in women) to $134 \mu \mathrm{g}$ per day (in men) [11]. In plasma and serum the selenium level varies from country to country that is much lower in Europe than Canada, Venezuela, Japan, and USA. In New Zealand intake of Se has been improved after the use Australian wheat rich in selenium [12] because selenium has many health benefits due to its anti-oxidative and anti-inflammatory properties. It also prevents bone resorption in disorders like osteoporosis. Recently, SeNPs are gaining more attention due to their low toxicity and excellent biological activities [13]. In this review we have discussed that how selenium therapy can prove to be effective for treatment of many diseases.

\section{Significance of Selenium to Human Health}

Selenium supplementation has positive impact on Human health. Many studies have shown importance of selenium in normal immune functions. Supplementation of selenium (400 g/day) to elderly volunteers in Arizona increased T cell count. Increase in CD4+ T cells subset was observed along with increased cytotoxicity of natural killer cells (NKCs) [14]. Supplementation of selenium in form of sodium selenite to head and neck cancer patients during radiation and surgery led to improved cell mediated immune responses [15]. Selenoproteins are important for normal function of activated $\mathrm{T}$ cells because $\mathrm{T}$ cells are sensitive to ROS and due to deficiency of selenoproteins involved in decreasing ROS they cannot proliferate in response to $\mathrm{T}$ cell receptor stimulation [16]. Selenium is also important for normal functioning of brain. Its depletion cause irreversible brain injury. Selenium is delivered to brain by selenoprotein $\mathrm{P}$ by binding to apoER2 a member of the lipoprotein-receptor family. Selenoprotein $\mathrm{P}$ improve neuronal survival and prevent cell death caused by beta-amyloid accumulation [17]. A study suggests that nanoparticles of selenium in size range of $5-15 \mathrm{~nm}$ depleted amyloid $\beta(\mathrm{A} \beta)$ plaque accumulation in brain by decreasing ROS production and appeared as a potential therapeutic agent for the treatment of Alzheimer's disease (AD) [18]. In addition, selenium is crucial in fertility, reproduction, normal thyroid hormone production and in prevention of cancer and type 2 diabetes [9]. SeNPs delivered in liposomes showed antidiabetic potential by preserving

*Corresponding author: Attya Bhatti, National University of Sciences and Technology (NUST), Sector H-12, Islamabad, Pakistan, Tel: 03352569369; E-mail: attyabhatti@asab.nust.edu.pk

Received: January 04, 2017; Accepted: February 19, 2018; Published: February 23, 2018

Citation: Majeed W, Zafar M, Bhatti A, John P (2018) Therapeutic Potential of Selenium Nanoparticles. J Nanomed Nanotechnol 9: 487 doi: 10.4172/2157 7439.1000487

Copyright: $\odot 2018$ Majeed W, et al. This is an open-access article distributed under the terms of the Creative Commons Attribution License, which permits unrestricted use, distribution, and reproduction in any medium, provided the original author and source are credited. 
the integrity of pancreatic $\beta$ cells with increase in insulin secretion, decrease of oxidative stress inhibition of inflammation in pancreas [19]. One human study also showed the significant antiviral effects of selenium supplementation (Figure 1) [20].

\section{Role of Se in Reducing Oxidative Stress}

The imbalance between the generation and detoxification or neutralization of the free radicals produced as a result of aerobic metabolism by the body is referred as oxidative stress [21]. In living systems the most important class of free radicles are ROS and RNS. The free radicals derived from oxygen are superoxide radical, peroxyl radical, perhydroxyl radical, hydroxyl radical and non-free radical species such as hydrogen peroxide and singlet oxygen, while the reactive nitrogen species include nitric oxide, nitrogen dioxide and peroxynitrite [22]. These reactive species are highly unstable because they contain one or more unpaired electrons in their outermost shell and remove electrons from other compounds to attain stability leading to chain reaction cascade generating more reactive species [23]. It has been found that in physiological system high level of ROS exert oxidative stress that leads to many diseases by damaging lipids, proteins and DNA [24]. To protect against the oxidative stress, living systems have a set of antioxidant enzymes such as glutathione peroxidase $(\mathrm{GPx})$, thioredoxin reductase (TrxR) and iodothyronine deiodinases (IDD) and selenium is main component of these antioxidant enzymes [25].Therefore its deficiency can lead to many chronic diseases.

\section{Role of Se in Reducing Inflammation}

Many studies have shown that selenium can reduce inflammatory response in body caused due to auto-immune disorders or inflammatory diseases. Inflammation is the normal response of the living body to any injury leading to the accumulation of body fluids and white blood cells along with release of prostaglandins and many inflammatory mediators [26]. NFkB pathway is involved in activation of inflammatory responses by enhancing the expression of many proinflammatory cytokines such as IL- 6 and TNF alpha. A recent study revealed significant decrease in expression of these pro-inflammatory cytokines when selenium as supplement was given because Se incorporates into selenoproteins and significantly increase their antioxidant function leading to decrease in inflammatory responses in autoimmune and inflammatory diseases [27]. The major problem regarding use of selenium is its bioavailability and toxicity. Therefore researchers have moved towards use of selenium as the nanoparticles. NPs have entirely different properties than bulk form of that same compound [28]. A recent study on inflammation induced irradiated rats showed that in addition to antioxidant activity nano selenium also decreases the expression of genes of pro inflammatory mediators like TNF- $\alpha$, PGE2 and TBAR [29]. Thus SeNPs are also effective in reducing inflammation.

\section{Effect of Se on Bone Health}

Studies related to selenium status in humans showed that $\mathrm{Se}$ concentration in plasma has an inverse relation with bone turnover rate [30]. In a case control study it was revealed that consumption of selenium, $\beta$-carotene and vitamin $\mathrm{E}$ had an inverse association with risk of hip fracture in elder smokers [31]. In patients of osteoporosis the activity of the enzyme Glutathione peroxidase (GPx) was found lower [32] which is the most abundant selenoprotein in mammals and protects cells from damage caused by oxidative stress. It reduces $\mathrm{H}_{2} \mathrm{O}_{2}$ into $\mathrm{H}_{2} \mathrm{O}$ using glutathione otherwise the increased level of $\mathrm{H}_{2} \mathrm{O}_{2}$ can damage cellular components [33]. Some previous studies showed that $\mathrm{Na}_{2} \mathrm{SeO}_{3}$ inhibited differentiation of osteoclasts by decreasing ROS production and also induced apoptosis in these cells by mitochondrial pathway [34] as high level of ROS stimulates osteoclast differentiation and maturation [35]. Increased activity of osteoclasts makes bone weaker by decreasing bone mass and lowering bone mineral density and can also lead to osteoporosis [32]. In another study it was revealed that in second generation selenium deficient male rats growth was retarded and also caused osteopenia and disturbed bone metabolism [36]. In a performed study pre-treatment with selenite prevented the adverse effect of $\mathrm{H}_{2} \mathrm{O}_{2}$ on induced inhibition of osteoblastic differentiation of MSCs. Moreover, it also suppressed the activation of ERK (extracellular signal-regulated kinase). Selenite pre-treatment also increased the gene expression and activity of GPx and reduced glutathione and lipid peroxidation in treated bone marrow stromal cells. This shows inverse relation of selenium status with risk of osteoporosis [37].

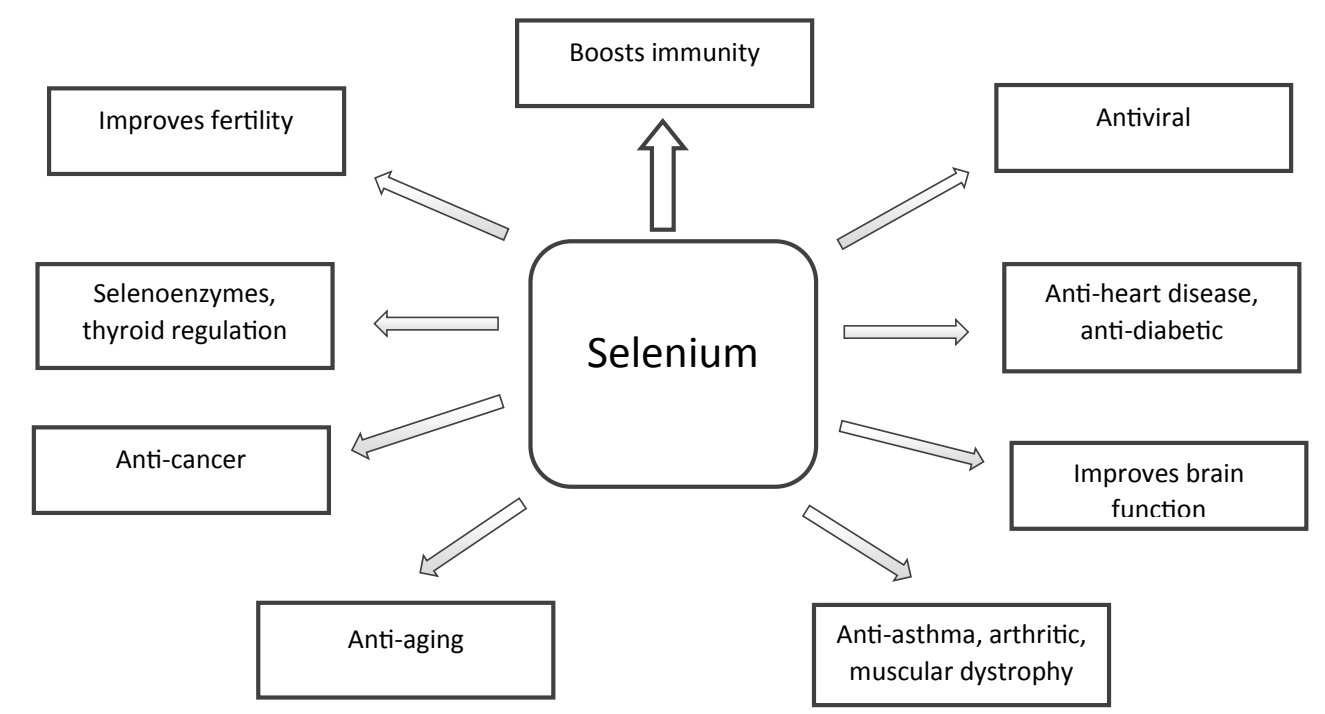

Figure 1: Health benefits of Selenium. 


\section{Therapeutic Efficacy of Se Based Nanoparticles}

No doubt selenium is an important trace element but it causes toxicity at level not much greater than the level required for its beneficial effects [38]. Therefore, research is going on nanoparticles of selenium for lowering its toxicity. Nanotechnology is the branch of science that focuses on understanding and controlling the objects at Nano scale. The prefix 'Nano' refers to $10^{-9}$ or one billionth part of any matter. Its concept was proposed by well known Richard P Feynman in meeting of American Physical Society in 1959 during his lecture " there's plenty of room in bottom"[39]. A lot of research is carried on diagnostic and therapeutic agents based on nanoparticles to treat pain, asthma, allergy, infections and different diseases like cancer, Alzheimer, diabetes etc. On Nano scale many fundamental properties of materials such as solubility, drug diffusion and release, half-life in blood and immunogenicity can be modified [40]. The best example is of antibodyconjugated quantum dots that are useful for disease detection as they show promising effects in targeting of in-vivo systems. Both Quantum Dots and liposomes of nano size are more effective than conventional drugs that have less directed delivery protocols [41]. Selenium in nano form has also shown excellent biological activities [42,43]. In a research it was revealed that SeNPs down-regulate mRNA expression of pro-inflammatory cytokines including inducible NO synthase (iNOS), interleukin IL-1 and TNF- $\alpha$ thus reducing inflammation [44]. SeNPs-SPS complexes made by decorating SeNPs with water-soluble derivative of Ganoderma lucidum polysaccharides (SPS) also have the potential to decrease inflammation by inhibiting NF- $k$ B, JNK $1 / 2$ and p38 MAPKs activation partially [44]. SeNPs can increase the activity of selenoenzymes with equal efficacy causing less toxicity to the cells as compared to selenite, Se-methylselenocysteine and selenomethionine [45]. Bioavailability, toxicity and antioxidant activity of selenium depends on its chemical form. Selenomethionine is the most widely used form of Se in food and supplements, though it has less toxicity and excellent bioavailability but some reports show that its excessive use can lead to toxic effects [46]. Therefore, instead of selenomethionine NanoSe can be used as its effect on upregulating glutathione peroxidase and thioredoxin reductase is comparable with selenomethionine with much lower toxicity [38]. Additionally, Nano-Se is a better chemopreventive agent compared with Se-methylselenocysteine [43]. In a study performed in $2015 \mathrm{SeNP}$ at concentration of $250 \mathrm{mg} / \mathrm{kg}$ b.w. was given and results showed that SeNPs acted as potent anti-inflammatory agent and significantly reduced arthritis induced parameters [47]. SeNPs are also used in cancer drug and gene delivery systems displaying combined effect of Se with the therapeutic drug cargo thus improving their anticancer activity [48].

\section{Conclusion}

Selenium is an essential micronutrient as it is required by many selenoproteins for their proper functioning. Due to importance of Se in reducing inflammation, decreasing oxidative stress and in improving bone health it is necessary to maintain its level in the body. Se supplementation trials in patients of RA showed beneficial effects but due to toxicity of Se in bulk form SeNPs are gaining more attention by the scientists. Nanoparticles of Se are less toxic and have some advanced properties in terms of diffusion, solubility and immunogenicity but chemicals used to reduce selenium salts can be harmful to patients, therefore, improvements are needed to make Se therapy more effective and safe.

More influence should be given on biological synthesis methods of selenium nanoparticles as an alternative to chemical and physical methods because green synthesis of nanoparticles is more economical, safe and eco-friendly. Nanoparticles produced as a result of this method are less toxic and safe to use of living organisms. Se NPs can also be used as micronutrient and as an alternative to antioxidant and anti-inflammatory drugs. These nanoparticles may help in providing new diagnostic techniques and systems for early diagnosis of diseases. Tissue repairing systems and targeted drug delivery systems can also be improved using Se NPs. The future outcomes of Se NPs seem to be very promising in increasing the efficacy of existing treatments and development of new therapies.

\section{References}

1. Rayman MP (2008) Food-chain selenium and human health: emphasis on intake. Br J Nutr 100: 254-268.

2. Kryukov GV, Castellano S, Novoselov SV, Lobanov AV, Zehtab O, et al. (2003) Characterization of mammalian selenoproteomes. Science 300: 1439-1443.

3. Bellinger FP, Raman AV, Reeves MA, Berry MJ (2009) Regulation and function of selenoproteins in human disease. Biochem J 422: 11-22.

4. Honkanen V, Konttinen YT, Sorsa T, Hukkanen M, Kemppinen $P$, et al. (1991) Serum zinc, copper and selenium in rheumatoid arthritis. J Trace Elem Electrolytes Health Dis 5: 261-263.

5. Yazar M, Sarban S, Kocyigit A, Isikan U (2005) Synovial fluid and plasma selenium, copper, zinc, and iron concentrations in patients with rheumatoid arthritis and osteoarthritis. Biol Trace Elem Res 106: 123-132.

6. Aletaha D, Neogi T, Silman AJ, Funovits J, Felson DT, et al. (2010) 2010 rheumatoid arthritis classification criteria: an American College of Rheumatology/European League Against Rheumatism collaborative initiative. Arthritis Rheum 62: 2569-2581.

7. Gibofsky A (2012) Overview of epidemiology, pathophysiology, and diagnosis of rheumatoid arthritis. Am J Manag Care 18: S295-302.

8. Thomson W, Barton A, Ke X, Eyre S, Hinks A, et al. (2007) Rheumatoid arthritis association at 6q23. Nat Genet 39: 1431-1433.

9. Rayman MP (2012) Selenium and human health. Lancet 379: 1256-1268.

10. Rayman MP, Infante HG, Sargent M (2008) Food-chain selenium and human health: spotlight on speciation. Br J Nutr 100: 238-253.

11. Rayman MP, Combs GF, Waters DJ (2009) Selenium and vitamin E supplementation for cancer prevention. Jama 301: 1876-1877.

12. Rayman MP (2004) The use of high-selenium yeast to raise selenium status: how does it measure up? Br J Nutr 92: 557-573.

13. Skalickova S, Milosavljevic V, Cihalova K, Horky P, Richtera L, et al. (2017) Selenium nanoparticles as a nutritional supplement. Nutrition 33: 83-90.

14. Wood SM, Beckham C, Yosioka A, Darban H, Watson RR (2000) $\beta$-Carotene and selenium supplementation enhances immune response in aged humans. Integr Med 2: 85-92.

15. Kiremidjian-Schumacher L, Roy M, Glickman R, Schneider K, Rothstein S, et al. (2000) Selenium and immunocompetence in patients with head and neck cancer. Biol Trace Elem Res 73: 97-111.

16. Carlson BA, Yoo M-H, Shrimali RK, Irons R, Gladyshev VN, et al. (2010) Role of selenium-containing proteins in T-cell and macrophage function. Proc Nutr Soc 69: $300-310$

17. Burk RF, Hill KE (2009) Selenoprotein P-expression, functions, and roles in mammals. Biochim Biophys Acta (BBA)-General Subjects 1790: 1441-1447.

18. Nazıroğlu M, Muhamad S, Pecze L (2017) Nanoparticles as potential clinical therapeutic agents in Alzheimer's disease: Focus on selenium nanoparticles. Expert Rev Clin Pharmacol 10: 773-782.

19. Ahmed HH, El-Maksoud MDA, Moneim AEA, Aglan HA (2017) Pre-clinical study for the antidiabetic potential of selenium nanoparticles. Biol Trace Elem Res 177: 267-280.

20. Montgomery JB, Wichtel JJ, Wichtel MG, McNiven MA, McClure JT, et al. (2012) Effects of selenium source on measures of selenium status and immune function in horses. Can J Vet Res 76: 281-291. 
Citation: Majeed W, Zafar M, Bhatti A, John P (2018) Therapeutic Potential of Selenium Nanoparticles. J Nanomed Nanotechnol 9: 487. doi: 10.4172/2157-7439.1000487

21. Quiñonez-Flores CM, González-Chávez SA, Del Río Nájera D, Pacheco-Tena C (2016) Oxidative stress relevance in the pathogenesis of the rheumatoid arthritis: a systematic review. BioMed res int 2016.

22. Phaniendra A, Jestadi DB, Periyasamy L (2015) Free radicals: properties, sources, targets, and their implication in various diseases. Indian $\mathrm{J}$ Clin Biochem 30: 11-26

23. Halliwell B, Gutteridge JM (1990) [1] Role of free radicals and catalytic metal ions in human disease: an overview. Methods enzymol 186: 1-85.

24. Zhang H, Luo Y, Zhang W, He Y, Dai S, et al. (2007) Endothelial-specific expression of mitochondrial thioredoxin improves endothelial cell function and reduces atherosclerotic lesions. Am J Pathol 170: 1108-1120.

25. Tinggi $U$ (2008) Selenium: its role as antioxidant in human health. Environ Health Prev Med 13: 102-108.

26. Ricciotti E, FitzGerald GA (2011) Prostaglandins and inflammation. Arterioscler Thromb Vasc Biol 31: 986-1000.

27. Duntas L (2009) Selenium and inflammation: underlying anti-inflammatory mechanisms. Horm Metab Res 41: 443-447.

28. Albrecht MA, Evans CW, Raston CL (2006) Green chemistry and the health implications of nanoparticles. Green Chemistry 8: 417-432.

29. El-Ghazaly MA, Fadel N, Rashed E, El-Batal A, Kenawy S (2016) Antiinflammatory effect of selenium nanoparticles on the inflammation induced in irradiated rats. Can J Physiol Pharmacol 95: 101-110.

30. Hoeg A, Gogakos A, Murphy E, Mueller S, Köhrle J, et al. (2012) Bone turnover and bone mineral density are independently related to selenium status in healthy euthyroid postmenopausal women. J Clin Endocrinol Metab 97: 40614070.

31. Zhang J, Munger RG, West NA, Cutler DR, Wengreen $\mathrm{HJ}$, et al. (2006) Antioxidant intake and risk of osteoporotic hip fracture in Utah: an effect modified by smoking status. Am J Epidemiol 163: 9-17.

32. Manolagas SC, Jilka RL (1995) Bone marrow, cytokines, and bone remodelingemerging insights into the pathophysiology of osteoporosis. N Engl J Med 332 305-311.

33. Rayman MP (2000) The importance of selenium to human health. Lancet 356 : 233-241.

34. Chung YW, Kim TS, Lee SY, Lee SH, Choi Y, et al. (2006) Selenite-induced apoptosis of osteoclasts mediated by the mitochondrial pathway. Toxicol Lett 160: $143-150$.

35. Steinbeck MJ, Kim JK, Trudeau MJ, Hauschka PV, Karnovsky MJ (1998) Involvement of hydrogen peroxide in the differentiation of clonal HD-11EM cells into osteoclast-like cells. J Cell Physiol 176: 574-587.
36. Moreno-Reyes R, Egrise D, Neve J, Pasteels JL, Schoutens A (2001) Selenium Deficiency-Induced Growth Retardation Is Associated with an Impaired Bone Metabolism and Osteopenia. J Bone Miner Res 16: 1556-1563.

37. Liu H, Bian W, Liu S, Huang K (2012) Selenium protects bone marrow stroma cells against hydrogen peroxide-induced inhibition of osteoblastic differentiation by suppressing oxidative stress and ERK signaling pathway. Biol Trace Elem Res 150: 441-450.

38. Wang $\mathrm{H}$, Zhang J, Yu $\mathrm{H}$ (2007) Elemental selenium at nano size possesses lower toxicity without compromising the fundamental effect on selenoenzymes: comparison with selenomethionine in mice. Free Radic Biol Med 42: 1524 1533.

39. Junk A, Riess F (2006) From an Idea to a Vision: There's Plenty of Room at the Bottom. Am J Phys 74: 825-830.

40. Zhang L, Gu F, Chan J, Wang A, Langer R, et al. (2008) Nanoparticles in medicine: therapeutic applications and developments. Clin Pharmacol Ther 83 761-769.

41. Kawasaki ES, Player A (2005) Nanotechnology, nanomedicine, and the development of new, effective therapies for cancer. Nanomedicine 1: 101-109.

42. Shi L, Xun W, Yue W, Zhang C, Ren Y, et al. (2011) Effect of sodium selenite, Se-yeast and nano-elemental selenium on growth performance, Se concentration and antioxidant status in growing male goats. Small Ruminant Res 96: 49-52.

43. Zhang J, Wang X, Xu T (2007) Elemental selenium at nano size (Nano-Se) as a potential chemopreventive agent with reduced risk of selenium toxicity: comparison with se-methylselenocysteine in mice. Toxicol Sci 101: 22-31.

44. Wang J, Zhang Y, Yuan Y, Yue T (2014) Immunomodulatory of selenium nanoparticles decorated by sulfated Ganoderma lucidum polysaccharides. Food Chem Toxicol 68: 183-189.

45. Bai K, Hong B, He J, Hong Z, Tan R (2017) Preparation and antioxidant properties of selenium nanoparticles-loaded chitosan microspheres. Int Nanomedicine 12: 4527.

46. Plateau P, Saveanu C, Lestini R, Dauplais M, Decourty L, et al. (2017) Exposure to selenomethionine causes selenocysteine misincorporation and protein aggregation in Saccharomyces cerevisiae. Sci Rep 7: 44761.

47. Malhotra S, Welling M, Mantri S, Desai K (2016) In vitro and in vivo antioxidant, cytotoxic, and anti-chronic inflammatory arthritic effect of selenium nanoparticles. J Biomed Mater Res B Appl Biomater 104: 993-1003.

48. Maiyo F, Singh M (2017) Selenium nanoparticles: potential in cancer gene and drug delivery. Nanomedicine 12: 1075-1089. 